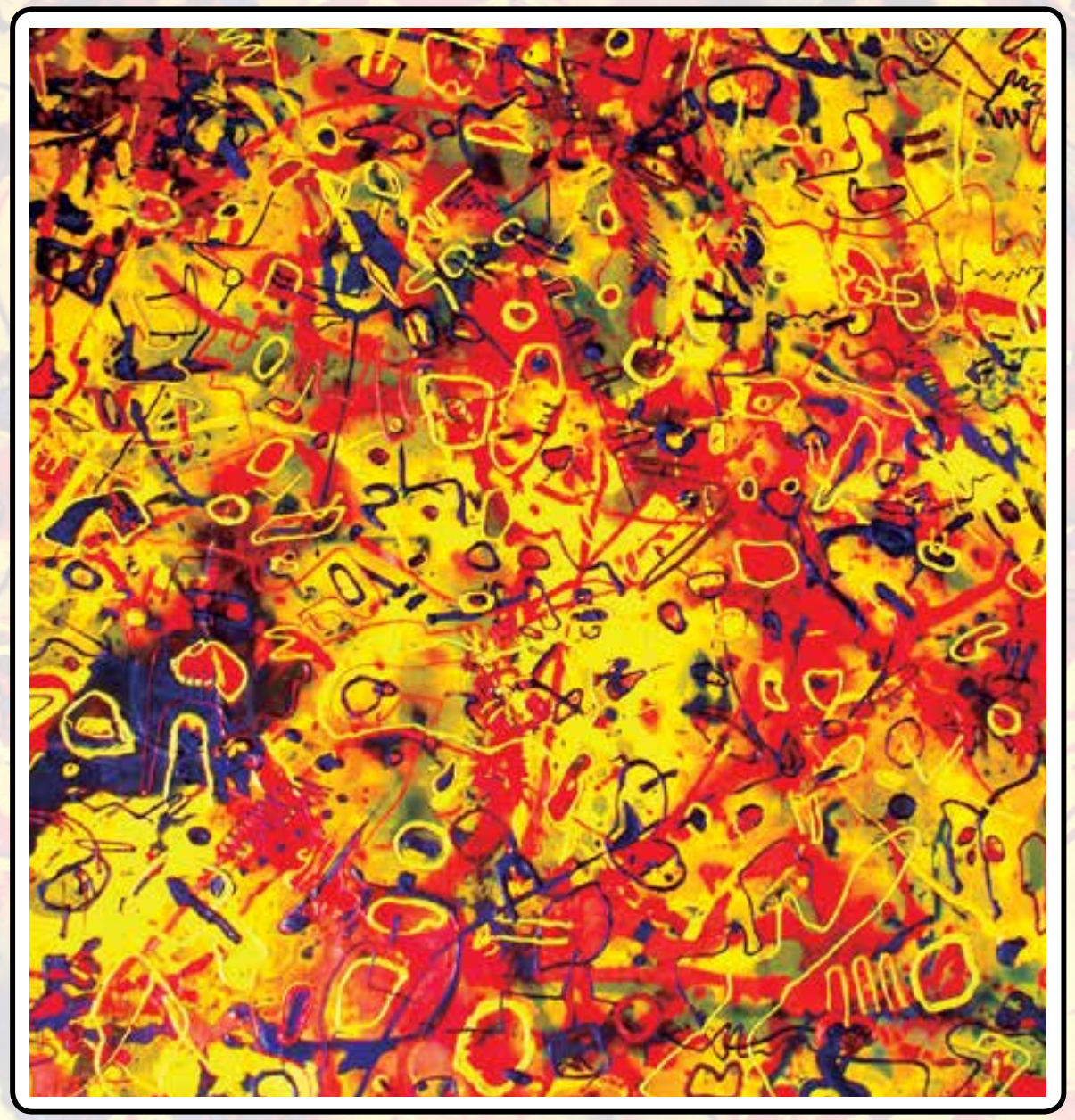

Camilo Umaña V.

Sin título

Acrílico sobre Lienzo 250 x 120 



\title{
ESTRATEGIA EXPORTADORA Y COMPORTAMIENTO INTERNACIONAL - ESTADO DE LA CUESTIÓN -
}

\author{
EXPORT STRATEGY AND INTERNATIONAL PERFORMANCE \\ -A STATE OF THE ART-
}

Diego A. Arenas $V^{I}$

\begin{abstract}
Resumen
Ante el inminente proceso de globalización económica que avanza con fuerza desde las últimas décadas del siglo pasado, las organizaciones han tenido que enfrentar retos crecientes y buscar estrategias que les permitan insertarse positivamente en el nuevo escenario mundial. Estudios recientes muestran, sin embargo, resultados divergentes en términos de eficacia de esas estrategias, por lo que es necesario realizar análisis comparados que permitan establecer los determinantes de su éxito o fracaso. El artículo refleja el ejercicio de revisión de la literatura reciente sobre el tema, como primera fase de una investigación que tiene como objetivo relacionar las estrategias de marketing y el comportamiento exportador de las PYMES de dos regiones del país: Santander y Antioquia.
\end{abstract}

\section{Palabras clave}

Estrategia exportadora, internacionalización, marketing internacional

\section{Clasificación JEL: L10}

\begin{abstract}
Faced with the imminent strongly economic globalization progresses since the last decades of the last century, organizations have faced increasing challenges and sought strategies to successfully enter the new world. However, recent studies show divergent results in terms of effectiveness of these strategies, so it is necessary to perform comparative analysis that establishes the causes of their success or failure. The article reflects the exercise of reviewing the recent literature on the subject, as the first phase of research that aims to link the marketing strategies and export behavior of SMEs in two regions: Santander and Antioquia. Given the proliferation of publications that have contributed to the debate in recent years, it is a conceptual and contextual approach to the subject of study.
\end{abstract}

\section{Key words}

Export strategy, internationalization, international marketing

\footnotetext{
1 Administrador de Negocios EAFIT, MBA con énfasis en Mercadeo University of Queensland, Australia. Profesor Facultad de Administración de Negocios Internacionales Universidad Pontificia Bolivariana, Bucaramanga (Colombia). Grupo de Investigación GRICANI. Correo electrónico: darenas80@hotmail.com
} 


\section{Introducción}

A partir de los años setenta del siglo pasado, la globalización de la economía como fenómeno que define el entorno mundial y la internacionalización como estrategia empresarial determinante para acompasarse a las profundas transformaciones del mismo, han estado en el centro de atención de los debates académicos, sin que existan aún acuerdos sobre sus alcances y factores determinantes. Para este estudio interesa destacar algunas manifestaciones del proceso, la primera de las cuales tiene que ver con la multiplicación de los flujos de comercio internacional, que, según el FMI, han crecido en términos reales a una tasa media de $6 \%$, superior al incremento promedio de la renta real mundial de los últimos años. El segundo fenómeno interesante es la transformación geopolítica provocada por el crecimiento de gigantes como China y la India y por la aparición -como protagonistas- de los países de reciente industrialización como Malasia, Tailandia, Corea, Singapur y Brasil, entre otros (Castells, 2001, 2006 Ohmae, 1990). Para reforzar esta idea, es interesante citar al analista japonés Ohmae, quien caracteriza los profundos cambios sufridos en el escenario mundial y sugiere la necesidad de una recomposición del actuar organizacional al afirmar que:

"A lo largo de las dos últimas décadas, el mundo ha cambiado de manera sustancial. Las reglas económicas, financieras, políticas, sociales, corporativas y personales ahora vigentes guardan muy escasa relación con las que regían veinte años atrás. Tiempos diferentes exigen un nuevo guión" (Ohmae, 2005: 9).

Este escenario de crecientes interrelaciones se hace cada día más complejo y convierte las estrategias de inserción internacional en una cuestión de supervivencia para las unidades de producción de países como el nuestro, relativamente rezagadas de las tendencias observadas en el resto del mundo. En la mayoría de los países, incluido Colombia, se han formulado políticas que pretenden conjugar la iniciativa privada y los estímulos del Estado para lograr una inserción favorable en los complejos escenarios actuales (DNP, 2008). Sin embargo, es preciso afirmar que los resultados de estos esfuerzos no resultan alentadores para algunas regiones, que parecen no haber realizado el "despegue" necesario para encaminarse con pasos seguros por el sendero de la mundialización.

Ante este panorama desigual se ha despertado un interés creciente en las comunidades académicas por explicar los determinantes de la internacionalización de las empresas y someter a contrastación los enfoques conceptuales a través de estudios empíricos. Así, a las teorías convencionales del comercio internacional, se han sumado en los últimos tiempos múltiples tendencias explicativas. Este esfuerzo -realizado casi siempre desde una perspectiva interdisciplinar y a partir de fundamentos conceptuales y formas de medición muy variadas- ha multiplicado la literatura sobre el tema hasta el punto que, quienes se acercan por primera vez 
a la problemática, encuentran casi imposible establecer con claridad los puntos de acuerdo y las divergencias entre sus estudiosos más reconocidos. Pero lo que más preocupa a quienes intentan derivar de las investigaciones algún tipo de orientación para las prácticas de las empresas nacionales y locales en su interés por incursionar con éxito en los mercados internacionales, es la fragilidad de las conclusiones y la falta de análisis comparativo cuando se trata de comprender realidades tan particulares como las que enfrentamos en la mayoría de los espacios territoriales de nuestro país. Esta preocupación está en el origen de un proyecto de investigación que pretende trascender la simple descripción de un fenómeno para incursionar en una propuesta metodológica que permita hacer valoraciones comparadas entre distintas unidades empresariales.

El interrogante-problema que ha orientado la propuesta de investigación tiene que ver con la relación entre la estrategia de marketing y el comportamiento exportador de la empresa, entendido como el mecanismo más común de internacionalización en nuestro medio (Gómez y González 2011). Al momento de intentar definir una metodología para acercase al objeto de estudio, resultó evidente la necesidad de contextualizar alrededor de lo que se entiende por este tipo de estrategia y, adicionalmente, sopesar en términos relativos otro tipo de factores -tanto internos como externos a la empresa- que la literatura ha destacado como relevantes a la hora de explicar la inserción positiva en los mercados internacionales. Así las cosas, fue necesario ampliar la visión inicial para contemplar la posibilidad de estructurar un posible modelo de evaluación de los principales factores que inciden en el posicionamiento de las empresas en los mercados internacionales para, una vez encontradas unidades empresariales más o menos homogéneas en términos de la mayoría de estos factores, poder determinar el peso específico de la estrategia de entrada a esos mercados en el éxito o fracaso de la meta de internacionalización. Adicionalmente, fue clara la necesidad de equiparar la experiencia de la región santandereana con la de países con características económicas semejantes a las nuestras o con la de otros espacios nacionales, con el fin de superar el vacío analítico que se mencionó en el párrafo precedente. Se decidió, entonces, incluir la evaluación de experiencias externas a Colombia a través de estudios secundarios rigurosos y tomar como un punto de referencia para la comparación ínter-regional nacional el Departamento de Antioquia, en el que es posible ubicar algunos sectores estratégicos comunes con los del Departamento de Santander.

Con el propósito en mente de diseñar, en la primera fase del estudio, una herramienta metodológica que permita medir la influencia de factores determinantes en el comportamiento exportador de las empresas colombianas, se abordó el análisis de los enfoques conceptuales que han intentado interpretar este proceso, destacando aquellos que pueden ser aplicables a las características del sector productivo nacional, marcado por una amplia participación de las micro, las pequeñas y medianas empresas. Esto significa que se dejaron de lado aquellas perspectivas sustentadas en 
la experiencia de las grandes multinacionales de los países más desarrollados, cuyo proceso de internacionalización está marcado por la inversión directa en otros países. Se incluyó la de las llamadas born global o empresas nacidas con enfoque global, con el fin de no descartar ninguna experiencia que pueda ser ilustrativa para el caso de las dos regiones del país que se analizan.

La apuesta por una herramienta metodológica tiene como propósito fundamental que las conclusiones del análisis posibiliten el acercamiento del sector empresarial a casos exitosos de internacionalización. Por ese motivo, de forma paralela se realizó el balance de algunos estudios empíricos -la mayoría de ellos, aunque no todos, tomados del ámbito iberoamericano- que parten de uno u otro enfoque interpretativo, con el fin de asimilar experiencias previas en términos de uso de categorías, métodos de cuantificación y perspectivas de análisis de la información, todo ello con el propósito de acercarnos a la problemática planteada para la segunda fase de la investigación, esto es la comparación entre PYMES santandereanas y antioqueñas. Como puede observarse, el estudio es novedoso en nuestro medio pero pretende construirse a partir de las reflexiones conceptuales y de lo que se ha avanzado en otros contextos en términos de confrontaciones empíricas, ya que consideramos que, en un tema como este, sólo ese ir y venir entre el debate conceptual y la aplicación empírica en diversos entornos, nos puede acercar a una realidad en la que pueden darse elementos comunes pero que está marcada, a su vez, por algunas especificidades que merecen atención especial.

Se cree, con Fillis (2001), que este recorrido por los diversos enfoques permitirá detectar propuestas metodológicas y referentes conceptuales que se puedan aplicar a otras experiencias. El interés fundamental consiste, entonces, en evaluar el camino recorrido hasta el momento para plantear nuevos desarrollos investigativos y posibilidades de asesoría a las empresas, orientados a la definición de estrategias que les permitan jugar con resultados positivos en el concierto internacional.

El autor español Sánchez-Meca (1999: 173) plantea, con razón, que

"en una época caracterizada por el crecimiento exponencial de la producción científica, las denominadas revisiones de la investigación constituyen el eslabón imprescindible entre el quehacer científico del pasado y del futuro, estableciendo el punto de partida de nuevas investigaciones".

Esa revisión inicial, sin embargo, puede desbordar la capacidad interpretativa de los investigadores cuando se enfrentan al enorme caudal de información existente hoy en Internet. Conscientes de esa limitación, se optó por privilegiar los artículos encontrados en las publicaciones más reconocidas en el ámbito del marketing internacional y las Tesis Doctorales, por considerar que son trabajos rigurosos realizados con el acompañamiento de expertos en el tema y evaluados por tribunales también especializados y, dentro de ellas, las que hacían análisis exhaustivos de la literatura precedente, para evitar una duplicación innecesaria de esfuerzos. De 
esa forma, se construyó un Estado del Arte -cuyas líneas principales se presentan en este artículo- que permitió acotar el objeto de estudio y delimitar las posibles metodologías para abordarlo.

\section{La Internacionalización Empresarial en el Debate Académico}

Con el fin de iniciar la presentación, se citará en extenso a Villareal para destacar la importancia del ejercicio de delimitación conceptual que aquí se emprende:

"La internacionalización y la multinacionalización implican procesos dinámicos y complejos difíciles de caracterizar, y que, sin embargo, tanto en ámbitos profesionales como académicos, es necesario describir y analizar. En este sentido existe, hoy en día, un claro problema de falta de delimitación conceptual de los principales términos necesarios para comprender y explicar dichos fenómenos empresariales. La ambigüedad resultante del empleo de los mismos términos para significados relativamente diferentes y de distintos términos para expresar realidades idénticas dificulta la comunicación y la investigación sobre estos temas. Es necesario, por tanto, contar con una delimitación conceptual rigurosa y compartida de los principales términos que caracterizan esta nueva realidad empresarial" (Villareal, 2005 p. 56).

Y, consecuentemente, se acude también a su definición de internacionalización:

"como una estrategia corporativa de crecimiento por diversificación geográfica internacional, a través de un proceso evolutivo y dinámico de largo plazo que afecta gradualmente a las diferentes actividades de la cadena valor y a la estructura organizativa de la empresa, con un compromiso e implicación creciente de sus recursos y capacidades con el entorno internacional, y basado en un conocimiento aumentativo" (Villareal, 2005:58).

Igualmente, es importante destacar la importancia de considerar, tanto las variables internas a la empresa como los factores externos al proceso y, en ese sentido, se rescatan las contribuciones de autores como Alonso (1994) y Beamish (1990), a quienes Monreal considera los pioneros en una aproximación integral a la internacionalización (Monreal, 2009).

Las primeras aproximaciones al proceso de internacionalización empresarial se hicieron desde la óptica macroeconómica, estuvieron enmarcadas en las teorías convencionales del comercio internacional y se ocupaban fundamentalmente de explicar los determinantes y modalidades de la inversión directa en países extranjeros, propia de las grandes firmas multinacionales, sin considerar otras formas de acceso a los mercados externos (Hymer, 1976; Vernon, 1966). El alcance del esfuerzo de internacionalización se determina, de acuerdo con estas vertientes, por la relación entre ventajas comparativas y costos de producción. Esas expresiones conceptuales -que generalmente se agrupan en los llamados enfoques desde la perspectiva macroeconómica- estarán fuera del horizonte de análisis, dado que el sector productivo colombiano -y el santandereano en particular- se configura como 
un conjunto de pequeñas y medianas empresas (PYME), registra una buena cantidad de microempresas y muestra un número relativamente escaso de manifestaciones maduras de internacionalización basada en formas diferentes a la exportación. Tampoco se tendrá en cuenta la tradicional perspectiva de la ventaja competitiva de las naciones formulada por Porter (1990) por considerar que en ella no se insiste suficientemente en la propia capacidad que tiene la empresa para competir en los mercados externos $\mathrm{y}$, por lo tanto, no permite considerar el interés específico en las estrategias de marketing como uno de los condicionantes del comportamiento exportador de las empresas.

Por la misma razón mencionada atrás, excluimos del estudio la llamada Teoría Ecléctica de Dunning (2000) -que pretende explicar las razones por las cuales las empresas se internacionalizan mediante la inversión directa en el extranjero- y cuya principal fortaleza descansa en la integración en un sistema único de análisis de los aportes de las teorías de costos de transacción y las más tradicionales relacionadas con la localización y la dinámica del comercio internacional.

Con el propósito de utilizar un criterio de clasificación ya diseñado para analizar los enfoques conceptuales sobre internacionalización de las PYMES, se siguió la propuesta de Monreal (2009) quien considera, además de la perspectiva que se menciona en el párrafo anterior, otras tres vertientes explicativas: las que tienen en común la visión de la internacionalización como un proceso, las agrupadas dentro de la perspectiva estratégica y los nuevos enfoques que trabajan sobre las llamadas empresas bornglobals.

\section{La internacionalización como proceso}

Los enfoques agrupados en esta denominación parten de una concepción dinámica y consideran que, desde la decisión inicial de exportar, las empresas acumulan experiencia y aprendizajes valiosos que les permiten consolidar su posición y utilizar formas de penetración en los mercados cada vez más complejas. Así, la internacionalización deriva en un compromiso creciente con los mercados externos, condicionado por los cambios organizativos que experimenta la empresa a lo largo de su trayectoria exportadora.

La llamada teoría de Uppsala o gradualista es representativa de esta perspectiva conceptual y se fundamenta, según Monreal (2009)- quien sigue a Alonso (1994)- en una visión conductista de la empresa, la cual desarrolla estrategias para actuar en un entorno incierto en función de sus propias capacidades institucionales, de tal forma que avanza desde estrategias de bajo costo y bajo riesgo (como la exportación) a aquellas más complejas que suponen mayor riesgo y mayor compromiso de recursos. Planteada inicialmente por Johanson y Vahlne (1997), considera que el conocimiento y el compromiso con el mercado son los factores dinámicos determinantes para explicar el tránsito de las organizaciones por las distintas etapas del proceso, que van 
desde actividades irregulares de exportación hasta el establecimiento de unidades de producción en el extranjero. El conocimiento del mercado permitirá conocer la cultura, pautas de consumo y normas gubernamentales de los distintos paísesobjetivo, reducir la incertidumbre y, de esta forma, será factor decisivo para aumentar el porcentaje de recursos internos destinados a consolidar la estrategia exterior de la empresa. El compromiso expresa la cantidad de recursos que la organización invierte en la estrategia de internacionalización, incluidos los que se transfieren a los mercados externos. Autores como Rialp $(1997,1999)$ y Alonso (1993) han complementado esta perspectiva desde lo conceptual y numerosos estudios empíricos la han utilizado como referente. Para el caso de las empresas manufactureras santandereanas, un estudio previo de Gómez y González (2011), quienes también han utilizado el esquema propuesto por los analistas suecos, ha comprobado que una buena parte de las unidades exportadoras refleja un cierto estancamiento en la estrategia exportadora, sin que se observen políticas claras al interior de la organización que hagan prever el tránsito hacia las otras etapas contempladas por el modelo gradualista, como la consecución progresiva de subsidiarias de ventas y de producción en el extranjero.

La principal crítica que ha recibido esta propuesta es el carácter lineal y determinista de la explicación y la ausencia de una interpretación sobre las condiciones o factores que inciden en el tránsito entre cada una de las etapas. La comprobación adicional de casos en los que algunas organizaciones han enfrentado con éxito la inserción internacional sin que medien las formas más simples de entrada a los mercados es otro elemento que ha recibido atención de varios autores, especialmente de aquellos que han encontrado empresas bornglobals en distintos países como Ganitsky en Israel (1989) Chang (1992) en Taiwán y Jovell en los países nórdicos (Jovell, 1990) o los que, mediante el análisis de casos, han comprobado diversas trayectorias de vinculación con los mercados internacionales que no se ajustan al esquema sugerido por los analistas de Uppsala. El último autor precitado, presenta una cita textual de los iniciadores de esta explicación en la que intentan salirle adelante a las críticas y reafirmar que el valor de su interpretación se limita a las primeras etapas del proceso. Dicen Johanson y Wiedersheim-Paul (citados por Jovell, 1990 p. 58)

"No estamos tratando de explicar por qué las empresas comienzan a exportar, pero asumimos que, debido a la falta de conocimiento de los países extranjeros y la propensión de evitar la incertidumbre, las empresas comienza a exportar a países cercanos, conocidos o similares en relación a sus prácticas de negocios".

Sin embargo, esta respuesta de los autores no responde del todo a las inquietudes formuladas respecto a la definición del modelo como restrictivo, en cuanto no considera alternativas de internacionalización muy comunes en el momento actual tales como las licencias, las franquicias o las joint ventures (Rialp, 1999) o como demasiado general, en la perspectiva de Jovell (1990). 
Desde el punto de vista metodológico, se cuestionan los criterios utilizados por quienes siguen este modelo, para la clasificación de las empresas en las distintas fases de la internacionalización, los más comunes de los cuales han sido la intensidad exportadora (\% de exportaciones sobre ventas totales) y los años de experiencia en los mercados externos. Como puede observarse, la delimitación de una u otra etapa puede ser arbitraria, ya que resulta muy difícil establecer en dónde se inicia una fase cualitativamente diferente. Sin embargo, para el conjunto de los países de América Latina, estudios recientes coinciden en señalar la vigencia de la mayoría de los postulados de esta corriente a la hora de evaluar la evolución del proceso de internacionalización. Adicionalmente, es interesante anotar que esta perspectiva no resulta excluyente de otras que se mueven hoy en el medio académico sino que, por el contrario, puede complementar y enriquecer el análisis cuando se hace referencia a la fase inicial de participación en los mercados externos (Leonidou y Katsikeas, 2002).

No solamente los analistas de la Escuela de Uppsala concibieron la internacionalización como un proceso por etapas. Asumiendo diferentes factores como determinantes encontramos a autores como Cavusgil (1980, citado por Martínez Carazo, 2009), quien establece varias fases marcadas por decisiones directivas que van desde lo que denomina marketing doméstico hasta la inversión directa de recursos en el exterior y considera algunas variables, tanto internas como externas, que pueden incidir en el éxito de la estrategia. Según Eusebio (2001), las investigaciones del autor mencionado se orientaron a validar empíricamente el peso de esas variables centrándose, especialmente, en la actitud y el compromiso de los directivos ante la internacionalización. La utilidad de esta corriente analítica para el propósito de la investigación propuesta, es que se centra en el estudio de la exportación como el mecanismo básico de internacionalización de las pequeñas y medianas empresas. Y, aún cuando se originó en Estados Unidos, donde existe un entorno más favorable para el accionar internacional de las organizaciones, algunas de sus ideas centrales pueden adaptarse perfectamente a las características del sector productivo colombiano y santandereano.

Algunos estudiosos han considerado como motor de desarrollo de los distintos momentos de la internacionalización las innovaciones a las que se somete la empresa en el proceso, pero más en el ámbito gerencial y organizacional que desde el punto de vista tecnológico. Es el caso de Barret y Wilkinson (1986), Moon y Lee (1990) y Moini (1995) -citados todos Monreal (2009)- por mencionar sólo los más relevantes. Alonso y Donoso (1998) se inscriben dentro de esta línea, ya que consideran que la decisión de internacionalizarse generalmente está acompañada de la intención de innovar, en un sentido amplio y todo ello depende de la capacidad creativa de los directivos comprometidos con el proceso, quienes acumulan experiencia y conocimiento que se traducen en capacidades incrementales para la actividad competitiva internacional. Interesa destacar que, la casi totalidad de estos estudios considera determinantes, el estilo de dirección -por llamarlo de alguna manera - y 
las características organizacionales, a la hora de evaluar los factores que pueden inhibir o promover el éxito en la inserción en los mercados internacionales.

\section{La visión estratégica de la Internacionalización}

Dentro de esta perspectiva se mencionan aquellas explicaciones que consideran la internacionalización como una actividad consciente y estratégica de las empresas, orientada a la creación de valor y a la adecuación de los objetivos empresariales a las tendencias cambiantes del complejo entorno internacional actual. Es importante destacar en este campo dos vertientes, dado que algunos de sus principios pueden ser aplicables a la situación particular de nuestro país: el enfoque de redes y el de las capacidades organizacionales.

El enfoque de redes visualiza la internacionalización como un proceso en el cual se intensifican progresivamente las ínter-relaciones entre los distintos actores del mercado que aumentan, de esa manera, las capacidades organizacionales para competir en el exterior. La consideración de proveedores, clientes, socios comerciales, competidores y gobierno, entre otros, como agentes intervinientes, remite directamente a la capacidad de la gerencia para aprovechar las relaciones en el exterior con el fin de mejorar la posición competitiva de la empresa. Se asume que ésta requiere recursos crecientes que pueden estar en manos de otras organizaciones y pueden ser aprovechados y optimizados a través de las redes (Chetty y Blankenburg, 2000). Los autores precitados consideran que las redes de negocios incluyen, tanto las organizaciones formales como las relaciones cotidianas informales, incluidos los contactos personales que pueden abrir posibilidades de participación en mercados distintos al doméstico. Es conveniente remitirse a la exposición que hace Eusebio (2001, p. 41) sobre esta corriente para destacar el trabajo de sus iniciadores Johanson y Mattson quienes afirman que la posición de una firma en la red determina, en cada momento del tiempo, las posibilidades y los límites de su actuación en el exterior. Así, proponen una clasificación de las empresas, según la tipología y la intensidad de sus relaciones, que contempla desde las firmas con muy bajos niveles de internacionalización que operan en entornos igualmente lejanos de los mercados externos, pasando por aquellas con altos índices de relaciones externas que pueden considerarse solitarias porque actúan en contextos competitivos casi siempre domésticos, hasta llegar a las totalmente internacionalizadas que se mueven en un entorno con igual proyección. Lo interesante de esta perspectiva es la consideración de la reacción en cadena que resulta de la actuación de algunas empresas pioneras que, al intervenir en redes externas, propician el acceso a la información para todas las unidades del sector y reducen con ello, el riesgo asociado con la actividad internacional.

En sus momentos iniciales, se pensó que el modelo de redes podía ser aplicable sólo a las grandes multinacionales que integran sus actividades productivas y 
comerciales en el escenario global pero, sin embargo, la experiencia reciente ha demostrado que el dinamismo relacional de directivos interesados en promover la participación en el mercado internacional, puede ser un factor decisivo para explicar el éxito exportador de muchas pequeñas y medianas empresas en todo el mundo. Tal como plantea Galván Sánchez (2003 p. 127) “de esta forma, a través de las relaciones de redes, las pequeñas y medianas empresas serán capaces de superar sus limitaciones con respecto al tamaño, que muchas veces pueden identificarse como limitaciones al crecimiento" Algunos estudios, incluso, permiten concluir que la mayoría de los directivos privilegia las fuentes personales de información a la hora de ubicar oportunidades de negocios en el exterior. Los contactos surgidos en las ferias internacionales, por ejemplo, o experiencias concretas de trabajo en el exterior de algunos de los miembros de la organización e incluso, relaciones surgidas fuera del contexto laboral se muestran como relevantes. Nuevamente, se evidencia aquí la importancia de la visión gerencial y el diseño de estrategias orientadas en forma clara hacia los mercados externos, aspecto que es enfatizado por Ellis (2000), uno de los iniciadores de esta interpretación.

A su vez, para el enfoque de las capacidades organizacionales, según las palabras de Monreal, (2009, p. 3) "los factores clave que explican el desempeño de las empresas son los internos: sus recursos y capacidades". Según este autor, el concepto puede aplicarse de manera válida al accionar de las empresas enfrentadas a un entorno internacional marcado por la complejidad y la turbulencia que les exige adecuar sus estrategias a las tendencias relevantes del mismo. Los analistas coinciden en mostrar que las raíces de esta propuesta se encuentran en la economía evolucionista que, de la mano de Winter y Nelson, ha postulado que las empresas se adaptan a su entorno mediante un proceso de aprendizaje y sus postulados apuntan a refutar la idea central de Porter de una posición competitiva que está relacionada especialmente con la posición de la empresa en el mercado de productos. A diferencia de esto último, el enfoque considera que las organizaciones son heterogéneas, cuentan con diferentes dotaciones de recursos y de capacidades que se constituyen en activos intangibles irrepetibles e insustituibles que les otorgan ventajas a la hora de internacionalizarse. (Jiménez, 2008). Dos hipótesis básicas están en el centro de esta interpretación: de una parte, como ya se dijo, existen diferencias entre las empresas que se relacionan con los recursos estratégicos que manejen y, de otra, estas diferencias -que pueden concentrarse en knowhow técnico o capacidades directivas, entre otras- son relativamente estables e inciden en el comportamiento de las organizaciones.

Cuando se trata de explicar el éxito del proceso de internacionalización de las empresas a partir de este enfoque, se menciona el carácter de inimitable de los factores estratégicos (llamadas "competencias distintivas" por Penrose en su trabajo pionero de 1959), debido a que son el resultado de rutinas laborales y esquemas organizativos propios de la empresa, lo que permite consolidar las capacidades para competir internacionalmente e incide positivamente en su desempeño exportador. 
Para muchos, esta perspectiva permite complementar otras visiones sobre el proceso de internacionalización en la medida que destaca la habilidad organizacional para crear y transferir conocimiento $y$, de esa forma, construir ventajas competitivas duraderas.

Al hacer referencia a las categorías utilizadas por el enfoque de recursos y capacidades, Jacobsohn plantea: "para Wernerfelt (1984 p. 172), un recurso es "cualquier cosa que pueda ser considerada una fortaleza o una debilidad de una empresa dada", o más formalmente, "todos aquellos activos (tangibles e intangibles) que se vinculen a la empresa en forma semipermanente" (1984, p. 172); algunos ejemplos que menciona son: marcas, conocimiento tecnológico, personal habilidoso, contactos comerciales, maquinaria, procedimientos y capital, entre otros. Barney, a su vez, (1991, p. 101) define los recursos como "todos los activos, capacidades, procesos organizacionales, atributos de la firma, información y conocimiento, entre otros, controlados por la empresa, que le permiten concebir e implementar estrategias que mejoren su eficiencia y efectividad". Esta definición tiene en cuenta no sólo los recursos, sino las capacidades y se centra únicamente en aquellos recursos generadores de fortalezas para la organización" (Jacobsohn, 2004, p. 142). Durán Herrera (1994), por su parte, define las capacidades competitivas como aquellas habilidades desarrolladas para manejar el conocimiento (tecnológico y de procesos), para asumir la dirección y gestión empresarial, y le suma a ello la experiencia en mercados locales, como requisito previo para incursionar en contextos internacionales.

Para sintetizar, se trata de que las organizaciones desarrollen un conjunto de recursos y de capacidades -mediante un proceso de aprendizaje continuo y apropiación del conocimiento, la innovación organizacional y la experiencia generados en su interior- de tal forma que sean difíciles de imitar y se conviertan en un activo sostenible en el tiempo que genere ventajas competitivas y, en consecuencia, verdaderas ganancias en el contexto internacional. De acuerdo con Gómez y Valenzuela (1999), las capacidades se aprenden con la rutina y se acumulan continuamente, como resultado de un proceso de cooperación y coordinación de recursos que se convierte, en sí mismo, en un activo intangible valioso para la organización. En ese contexto, "las estrategias son concebidas como intentos de identificar, proteger y explotar las habilidades y recursos únicos a disposición de la empresa, con objeto de ganar ventaja competitiva en el mercado" (Jiménez, 2008 p. 6). Esta definición resulta muy adecuada al propósito del presente estudio de relacionar el desempeño exportador de las PYME con las estrategias de marketing, ya que permite evaluarlas en términos de eficiencia y eficacia para los objetivos de internacionalización.

Las principales críticas a esta perspectiva han surgido de Porter, quien cuestiona la incapacidad del modelo para incluir las variables del entorno como determinantes de la competitividad, crítica que incluso ha sido reconocida por algunos de sus más 
fieles adeptos. Lo importante de destacar, entonces, es que el enfoque de los recursos y capacidades, al enfatizar en los factores internos que explican el comportamiento empresarial en los mercados, puede resultar complementario de aquellos que contemplan la influencia de variables exógenas.

Por último, es necesario mencionar dentro de este grupo, el nuevo enfoque de las llamadas empresas born global surgido en los últimos años ante la evidencia, como se planteó atrás, que muchas empresas de creación reciente tienen una importante participación en los mercados internacionales y no han pasado por el proceso gradual predicho por algunas teorías, especialmente la de la Escuela de Uppsala. Las principales conclusiones de los estudios sobre estas empresas apuntan a definir un conjunto de competencias organizacionales que les dan ciertas ventajas competitivas en los mercados externos entre los que se destacan la flexibilidad, la posibilidad de adaptarse rápidamente a cambios en el entorno, tanto de la producción como de las ventas y, especialmente, el carácter emprendedor de sus fundadores (Galván, 2003; Iborra, Menguzzato y Ripollés 1988; Moen y Servais, 2002;Hashai y Almor, 2002; Gheorghiu, 2007). Los autores nombrados establecen algunos criterios para definir este tipo de empresas -que se tomarán en cuenta en el estudio- como una edad máxima de diez años (aunque para otros analistas veinte años también es un horizonte reciente) y un porcentaje de ventas en el exterior de, por lo menos, del $25 \%$ de su producción. El interés primordial será, por supuesto, analizar las estrategias de marketing de las que pueden considerarse exitosas, con el fin de construir un acumulado de experiencias orientadoras para el resto de las organizaciones.

Como la mundialización de la economía es un proceso dinámico que continuamente presenta cambios en el escenario internacional, tales como innovaciones tecnológicas $\mathrm{u}$ organizacionales, la presencia de nuevos actores protagónicos en el mercado o las alianzas entre esos actores y el incremento del talento humano formado en un contexto de permanente innovación, el debate respecto a los caminos que pueden adoptar las empresas para integrarse a esas transformaciones sigue vigente. Las últimas tendencias muestran la convicción de la mayoría de los analistas sobre el escaso poder explicativo de todos los enfoques que hemos analizado, cuando se utilizan de manera dogmática sin considerar las variables puestas en juego por las interpretaciones alternativas. De allí que sea posible observar en la literatura más reciente un intento por formular "modelos integradores" que superan esta visión unilateral y prometen convertirse en herramientas valiosas a la hora de orientar los estudios empíricos (Losada et al., 2006).

\section{Determinantes del Comportamiento Exportador}

En un artículo de hace unos años, sus autores presentaban el tema anunciando: "El estudio de los factores que influyen en el resultado exportador de las empresas es una de las líneas de investigación más prolíferas en la literatura de marketing 
internacional" (Losada Pérez et al., 2006, p 1). Han pasado seis años desde entonces y puede afirmarse, sin temor a equivocarse, que el interés aún crece, especialmente en lo que se refiere a la contrastación empírica de las formulaciones conceptuales iniciales. El interés fundamental en este apartado es presentar de forma paralela las principales vertientes interpretativas del fenómeno exportador -considerado como el camino más sencillo y con menor riesgo para lograr la internacionalización- y aquellas investigaciones que han adelantado conclusiones sobre su validez en casos específicos o, en otras palabras, han "testeado" el modelo conceptual. También aquí fue necesario un esfuerzo de integración y de acotación importante, con el propósito de buscar referentes válidos para la situación de nuestro país.

La clasificación de Aaby y Slater (1989) establece dos grandes categorías en los estudios: los que tienen su centro de atención en los rasgos del entorno como determinantes del proceso exportador y los que insisten en la importancia de las características y competencias organizacionales y en la estrategia, como principales factores explicativos. Para enlazar con el apartado anterior el énfasis se define, no en aquellas aproximaciones conceptuales del primer grupo, que privilegian los factores que condicionan la competitividad de los países o sectores en su conjunto, como los marcos institucionales y las condiciones del país de origen -dentro de las cuales el diamante de Porter ha sido, posiblemente, la más utilizada en los últimos años- sino en las que, desde una perspectiva microeconómica, resaltan el papel jugado por las características estructurales y organizativas de las empresas y los esquemas y estilos directivos que las presiden.

Diversos analistas coinciden en postular a Madsen (1987) Zou y Stan (1998) y Leonidou (2002) como los autores que han logrado una síntesis juiciosa de las principales tendencias investigativas respecto a los determinantes del comportamiento exportador y a Sousa (2004) y Sousa et al. (2008) como quienes han resumido las líneas del debate acerca de las posibilidades de medir ese comportamiento, ya que uno de los factores que genera confusión e inconsistencia entre los resultados obtenidos tiene que ver con la ausencia de indicadores estandarizados para evaluar el éxito en la actividad exportadora. Se intentará, en un primer momento, presentar las principales conclusiones de sus estudios para, posteriormente, acercarse a los autores que han avanzado en la formulación de una metodología integral, a partir de la cual se puedan emprender análisis comparativos como el que se tiene en mente.

Tal como se plantea atrás, un primer elemento para destacar como resultado de la lectura de los estudios empíricos mencionados, es la necesidad de contar con un concepto claro de "comportamiento exportador" (o performance que es el término anglosajón que se transfiere, sin traducción, en algunos documentos consultados como Losada Pérez et al., 2006) y que ha sido asimilado a "éxito exportador" o "intensidad exportadora". La conceptualización supone, adicionalmente, utilizar indicadores objetivos que permitan la medición de la actividad de las empresas en los mercados exteriores y posibiliten la evaluación comparada de comportamientos 
diferenciados. Lages y Sousa -ya citados- han avanzado considerablemente en términos de una propuesta metodológica en este sentido. En el examen analítico de Del Río y Valera (2005 p. 190) sobre los estudios más recientes se concluye que los indicadores más utilizados por los investigadores son

"intensidad exportadora (ratio "exportaciones/ventas totales"), cifra de ventas de exportación, dinamismo exportador (crecimiento de la intensidad exportadora), crecimiento de las ventas de exportación, rentabilidad de las exportaciones ${ }^{6}$ y satisfacción global con el rendimiento de las exportaciones".

Por tal motivo, serán los indicadores objetivos de intensidad exportadora y dinamismo en las exportaciones las que se utilicen como referentes para calificar el desempeño de las empresas estudiadas en el mercado exterior.

Respecto a los determinantes del comportamiento exportador se acoge la valoración de la literatura que realizó Monreal (2009) en su Tesis Doctoral, por considerar que presenta un panorama muy completo y bien sustentado en referencias bibliográficas, de las principales tendencias que se encuentran en el debate académico sobre el tema. Para ser consecuentes con los argumentos presentados en los apartados precedentes, se deja de lado la consideración sobre los factores externos explicativos del comportamiento exportador (política gubernamental, situación económica del país y características de la demanda, entre otros) para concentrarse en los determinantes internos y encauzar, de esa forma, el principal objetivo de la investigación. Monreal considera que existe un acuerdo casi generalizado entre los académicos respecto a que las características de la empresa -entre las que destaca su tamaño, la antigüedad, la experiencia internacional y la cultura organizativa-inciden de manera determinante en los esfuerzos exportadores. Le suma a estos factores las características objetivas de los directivos, como la edad, la formación y las habilidades para moverse en el contexto internacional y lo que llama las características subjetivas, dentro de las cuales contempla básicamente las percepciones y las actitudes para concluir que "la mayoría de trabajos respalda la proposición que es de esperar: las empresas cuyos directivos tienen una actitud positiva hacia la exportación son más propensas a la misma" (Monreal, 2009 p. 65). En consecuencia, y para ser coherente con las argumentaciones precedentes, se contemplarán indicadores subjetivos como la percepción de los directivos sobre los procesos adelantados por la empresa para enfrentarse a los retos de internacionalización.

Según el autor mencionado en el anterior párrafo, la mayoría de los estudios recientes propone como variable explicativa importante del desempeño exportador, además de las características de la organización y de sus directivos ya mencionadas, el diseño de la estrategia exportadora, que contempla la selección del tipo de estrategia competitiva que se seguirá para posicionarse en los mercados externos, la selección y fragmentación de los mercados y las variables estratégicas de marketing, entre las que menciona las políticas de precio, promoción y distribución. Como uno de los objetivos de la investigación en su segunda fase -además de la elaboración de un posible modelo integrador que contemple los principales determinantes del 
comportamiento exportador- se refiere al análisis de la estrategia de marketing de las empresas exportadoras, con el fin de establecer elementos comunes entre aquellas formuladas por las empresas que han podido acceder con éxito a los mercados internacionales, en el siguiente y último apartado del artículo, se hará referencia a estudios de interpretación y a confrontaciones empíricas sobre este tema en particular.

\section{Estrategia Exportadora y Desempeño Internacional}

De acuerdo con Del Río y Valera (2005, p. 183) quienes, a su vez, se basan en Leonidou (1995):

"En una economía crecientemente globalizada, en la que el entorno competitivo de las empresas se extiende más allá del mercado nacional, la exportación es la alternativa de entrada en los mercados internacionales más frecuente, ya que requiere un compromiso de recursos humanos, financieros y de otro tipo menor que otras opciones de internacionalización. Esto es especialmente cierto en el caso de las Pymes, dado que estas organizaciones, normalmente, cuentan con escasos recursos materiales y de conocimiento para abordar los mercados exteriores a través de fórmulas más arriesgadas desde el punto de vista del compromiso de recursos".

Por tanto, el estudio de las estrategias exportadoras exitosas -lo mismo que de aquellas que han resultado restrictivas e ineficientes- se convierte en una línea de interés para los académicos de marketing y los directivos empresariales.

Tal como afirman Flor Peris y Oltra Mestre (2009, p. 2)

"Respecto a la estrategia exportadora, la investigación sobre variables relacionadas con la estrategia de expansión de mercados y con el grado de adaptación del programa de marketing no ofrecen resultados concluyentes sobre qué enfoque favorece el éxito exportador".

En su concepto, para poder estudiar la efectividad o limitaciones de la estrategia diseñada con el fin de alcanzar los mercados internacionales, es preciso tener claridad sobre cuáles son los factores que la componen $\mathrm{y}$, especialmente, las interrelaciones entre ellos. Con ese propósito, es necesario un acercamiento a la literatura más destacada sobre el tema teniendo como referencia, también en este caso particular, las revisiones realizadas por autores que han "puesto a prueba" los diferentes enfoques doctrinales.

Una de las decisiones más importantes a las que se enfrentan los directivos en el proceso de internacionalización tiene que ver con la selección entre lo que la literatura especializada llama estrategia de "concentración" o de "diversificación", entendido por la primera el énfasis en la penetración a profundidad en unos pocos mercados para enfrentar, una vez consolidada la posición en éstos, una expansión 
hacia otros espacios territoriales. A diferencia de ello, la estrategia de diversificación tiene que ver con el intento de ampliar el radio de exportaciones aún cuando en ninguno de los mercados se penetre a profundidad (Losada y otros, 2007). Una gran cantidad de estudios ha intentado evaluar empíricamente los beneficios de una u otra opción, pero, podría decirse que se presenta un sorprendente equilibrio entre unos y otros, de tal forma que el resultado no es concluyente. Así, una primera conclusión a la que se llega después de este recorrido bibliográfico es que no existe una estrategia internacional que sea óptima en sí misma, ya que su éxito depende de las características de la empresa que exporta, del bien producido y del sector al que pertenecen (Flor Peris, 2010). Por tanto, se acotará el concepto de estrategia exportadora para hacer referencia, concretamente, a la estrategia de marketing.

El artículo de F M Moghaddam y otros (2011) “The Influence of Export Marketing Strategy Determinantson Firm Export Performance: A Review of Empirical literatures Between 1993-2010” publicado en el International Journal of Fundamental Psychology \& Social Sciences, facilitó el trabajo de acercarse conceptualmente al tema de estudio ya que, como el título lo menciona, es el resultado de un seguimiento exhaustivo a la literatura reciente sobre el tema y un ejercicio de síntesis muy útil para los propósitos del estudio. Así, se tomó como referencia su contribución en lo que se refiere a los componentes fundamentales de la estrategia de marketing que pueden afectar el comportamiento exportador de las empresas, que contempla la estrategia de precios, de promoción y publicidad, de distribución y de producto presentados en el siguiente cuadro:

\begin{tabular}{|c|c|}
\hline $\begin{array}{l}\text { ESTRATEGIA PRECIO EN MERCADEO } \\
\text { La ventaja del bajo costo, competitividad en el precio, ajuste de los precios } \\
\text { para exportar a mercados internacionales, método del precio, estrategia } \\
\text { de precios skimming, competitividad de precios, precio del producto, } \\
\text { estrategia de precio, términos de ventas, política de créditos, estrategias de } \\
\text { cambios de divisas y adaptación de precios. }\end{array}$ & \\
\hline $\begin{array}{l}\text { ESTRATEGIA PROMOCIÓN EN MERCADEO } \\
\text { Actividades de inversión en promoción, publicidad y promoción, agencias } \\
\text { comercial en mercados de exportación, intermediarios comerciales, gastos } \\
\text { de publicidad, adaptación a la promoción, publicidad, promoción de ventas, } \\
\text { personal de ventas y participación ferias. }\end{array}$ & 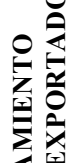 \\
\hline $\begin{array}{l}\text { ESTRATEGIA DISTRIBUCIÓN EN MERCADEO } \\
\text { Adaptación a los distribuidores, tipos de canales de exportación, estrategias } \\
\text { distribución y soporte a distribuidores internacionales, representantes de } \\
\text { ventas internacionales y compradores directos, centros principales, canales } \\
\text { exportación directo, canales distribución, disponibilidad. }\end{array}$ & $\sum_{0}^{\infty} \sum_{\substack{\infty \\
0}}^{\infty}$ \\
\hline $\begin{array}{l}\text { ESTRATEGIA PRODUCTO EN MERCADEO } \\
\text { Diseño de producto, mezcla de marca (nombre, señal, símbolos y diseño), } \\
\text { garantía, servicio al cliente antes y después de la venta, ventajas del } \\
\text { producto (lujo, prestigio y calidad), adaptación del producto y estrategia de } \\
\text { diseño, calidad y servicio, imagen de marca, diferenciación del producto, } \\
\text { diversificación de productos, innovación de productos, ventaja de marca, } \\
\text { empaque y etiquetas. }\end{array}$ & 牙 \\
\hline
\end{tabular}


El trabajo posterior consistirá en seleccionar las variables más adecuadas para aproximarse a la realidad del sector empresarial colombiano, teniendo siempre presente la posibilidad de contar con herramientas metodológicas -cuantitativas o cualitativas- para su operacionalización y manejo analítico.

\section{El "Estudio de Casos" como Propuesta Metodológica}

Como se plantea al inicio del documento, la gran cantidad de estudios encontrados sobre el tema puede desalentar -y angustiar- a un investigador que busca derroteros claros para enfrentar los interrogantes que dieron origen a su preocupación. Si esto es cierto para los enfoques conceptuales, lo es también cuando se trata de elegir un marco metodológico adecuado para alcanzar sus propósitos investigativos. Por esa razón, se tomaron como referencia los planteamientos de Villarreal (2006), en su investigación doctoral realizada sobre la estrategia de internacionalización de las empresas multinacionales vascas. En ella se fundamenta, con argumentos serios y consistentes, la adopción del estudio de casos como marco metodológico idóneo cuando se trata de analizar empresas pertenecientes a diferentes sectores y en las que no es fácil estandarizar los componentes de la estrategia para su análisis por métodos cuantitativos. Para Villarreal y Landeta,

"El estudio de casos permite analizar el fenómeno objeto de estudio en su contexto real, utilizando múltiples fuentes de evidencia, cuantitativas y/o cualitativas simultáneamente. Por otra parte, ello conlleva el empleo de abundante información subjetiva, la imposibilidad de aplicar la inferencia estadistica y una elevada influencia del juicio subjetivo del investigador en la selección e interpretación de la información. El estudio de casos es, por tanto, una metodología de investigación cualitativa que tiene como principales debilidades sus limitaciones en la confiabilidad de sus resultados y en la generalización de sus conclusiones, lo que la enfrenta a los cánones científicos más tradicionales y lo que, de alguna manera, la ha marginado (que no excluido) frente a otras metodologías más cuantitativas y objetivas como metodología científica de investigación empírica" (Villarreal y Landeta, 2010 p. 32).

\section{A manera de conclusión}

Dos reflexiones para finalizar el documento. A pesar de la enorme cantidad de literatura que se ha producido en los últimos años sobre el tema de internacionalización de las organizaciones empresariales, no existen resultados concluyentes acerca de cuáles caminos y estrategias facilitan el proceso, por lo que se justifica plenamente mantener abierta esta línea de investigación y emprender la tarea de multiplicar los estudios empíricos que den luces sobre los rasgos diferenciadores del sector productivo de nuestro país y, fundamentalmente, de las razones que explican su rezago cuando se compara con otras experiencias cercanas. 
De allí que, después de este recorrido por marcos conceptuales y metodológicos y estudios de caso, se considera relevante reiterar la necesidad de formular una propuesta metodológica integradora que, permita acercarse a la definición de los factores determinantes del comportamiento exportador de las empresas en nuestro país. De otra parte, es urgente tender "puentes" efectivos entre los académicos y el sector empresarial, de tal forma que las investigaciones adelantadas se conviertan en insumo para que los directivos acumulen elementos de juicio suficientes para formular estrategias efectivas hacia la internacionalización.

En ese contexto, el estudio propone una metodología inicial para escoger las empresas que tenga en cuenta las variables que definen la internacionalización según el enfoque gradualista con el fin de que las unidades empresariales tengan un nivel de homogeneidad en términos de sus características productivas y su relación con el mercado exterior, para que pueda hacerse la comparación de manera objetiva. Adicionalmente, para la comparación considera un esquema metodológico que, aun a pesar de sustentarse en la teoría de los recursos y capacidades, permite también reconocer la influencia de las variables del entorno. Para ello, son dos las "capacidades" que se han de medir: la capacidad de los directivos de la empresa para reconocer las tendencias del entorno internacional y su capacidad para diseñar estrategias de marketing internacional -precio, promoción y distribución- que le permitan a la organización ganar progresivamente en los complejos escenarios internacionales. Mediante la confrontación de experiencias positivas y negativas en términos de internacionalización, se puede establecer el peso específico de estos dos factores en el comportamiento exportador como paso inicial de la internacionalización. En este esquema, entonces, se combinan referentes de varias de las vertientes mencionadas en el artículo y se hace un reconocimiento a los rasgos predominantes del sector empresarial objeto de estudio.

\section{REFERENCIAS}

Aaby, N-E.y Slater, S. (1989). Management influences on export performance: a review of the empirical literature 1978-1988. International Marketing Review, 6(4), 7-25

Aragón Sánchez A y Monreal Pérez J (sf) La estrategia como factor de internacionalización de la PYME española Revista Internacional de la Pequeña y Mediana Empresa. Vol.1. No. 1. (http://www.revistainternacionalpyme.org/ volumenes/volumenuno/vollnum1/articulos/pyme\%20art2.pdf) 02-21-2012

Arenas Gaitán, J, et al (2006) "La revisión meta-analítica de la literatura: una guía para la investigación en marketing" MKT-A - Teorías, Modelos y Metodologías en Marketing (http://www.anpad.org.br/ema/2006/dwn/ema2006-mkta-026.pdf) 03-16-2012 
Alonso, J.A. (1994), “El Proceso de Internacionalización de la Empresa”, Información Comercial Española (ICE), No. 725 (Enero), pp. 127-143

Alonso, J. A. (1993). “Capacidades exportadoras y estrategia internacional de la empresa” en Juan Valarde, José L. García Delgado y Andrés Pedreño (dir): "Empresas y empresarios españoles en la encrucijada de los noventa". Madrid: Ed. Civitas. Pp.:205 - 258.

Alonso, J. A. y Donoso, V (1996). “Obstáculos a la internacionalización y políticas públicas de promoción. El caso de España”. Papeles de economía española. No. 66. Pp. $124-143$.

Alonso J. A. y Donoso V (1997) Competir en el exterior. La empresa española y los mercados internacionales Madrid, Instituto Español de Comercio Exterior

Barney J. (1991) "Firm resources and sustained competitive advantage", Journal ofManagement, 17: 99-120

Beamish, P. W. (1990) The Internationalisation process for smaller Ontario firms: a research agenda. En: Research in global strategic management -international business research for the twenty-first century: Canada's new research agenda, (ed. A. M. Rugman), pp. 77-92, JAI PressInc, Greenwich.

Cabrera, M. y De Saá, P. (1996). La empresa familiar desde la perspectiva de la teoría de recursos y capacidades; Economía globalizada: Retos y cambios. Granda: AEDEM. (http://www.usc.es/ ideasusanaab.doc) 03-08-2012

Carneiro J., Da Rocha A. y Ferreira J. (2007) A Critical Analysis of Measurement Models of Export Performance. BAR, Vol. 4 No. 2, pp. 1-19 Mayo/Agosto (http:// www.anpad.org.br/bar ) 06-04-12

Castells M. (2001) La Era de la Información. México, Siglo XXI Editores.

Castells M. (2006) La Sociedad Red. Madrid Alianza Editorial

Calvo, J. (1993), "La Internacionalización de las PME Manufactureras Españolas", Economía Industrial, No. 291, Mayo-Junio, pp. 66-75

Cavusgil, S. T. y Nevin, J. R. (1981): “Internal determinants of export marketing behaviour: An empirical investigation", Journal of Marketing Research, 16 (febrero), pp. 114-119

Cruz Camacho M. A. y Osorio Uribe V. (2010) Comportamiento de las Empresas Colombianas en sus Procesos de Internacionalización. Trabajo de Grado para optar por el título de: Magíster en Administración Universidad ICESI, Cali. http:// www.icesi.edu.co/biblioteca_digital/bitstream/10906/5372/1/PG.pdf 
Cuervo García, A. (1993): "El papel de la empresa en la competitividad”, Papeles de Economía Española, 56,363-378.

Chetty S. y Blankenburg Holm D. "The Role of Business Networks in the Internationalisation of Manufacturing Firms: A Longitudinal Case Study" International Business Review, 2000, vol. 9, issue 1, pages 77-93

(http://econpapers.repec.org/article/eeeiburev/v_3a9_3ay_3a2000_3ai_3a1_3ap_ 3a77-93.htm )

Cruz M., P. A. y Calderón H., G. (2006) “Cambio y Generación de capacidades competitivas" Revista EAN, No. 5, 27-44

DNP (2008) Política Nacional de Competitividad y Productividad. Documento CONPES 3527, Bogotá.

Del Río Araújo, M. L. y Varela Neira, M. C. (2005) “Características del directivo responsable del área internacional y resultado exportador de las empresas industriales". Investigaciones Europeas de Dirección y Economía de la Empresa Vol. 11, No. 2 2005, pp. 183-207

Dunning, JH (2000) “The eclectic paradigm as an envelope for economic and business theories of MNE activity” International Business Review, Elsevier, Vol. 9 (2)

Durán Herrera, J. (1994). "Factores de competitividad en los procesos de internacionalización de la empresa”. ICE, Revista de Economía No. 735. Pp. 21 -41 .

Escandón Barbosa (2009) D.M. "Factores que inciden en la creación de born global en Colombia” Estudios Gerenciales Vol. 25 No. 113 (Octubre - Diciembre), 55-73 ICESI, Cali

Eusebio R. (2001) Los determinantes del resultado exportador: un análisis comparativo entre empresas españolas e italianas. Tesis Doctoral Universidad Autónoma de Barcelona, España (http://tdx.cesca.cat/handle/10803/3948?Show =full)

Eusebio, R., et al. (2004) "Los determinantes internos de la propensión exportadora: un análisis comparativo entre empresas españolas e italianas". Encuentro de profesores universitarios de Marketing, Universidad de Alicante, España (http:// www.epum2004.ua.es/aceptados/215.pdf ) 03-02-12

Fernández Moreno M, et al. (2008) "Factores determinantes del éxito exportador. El papel de la estrategia exportadora en las cooperativas agrarias" CIRIECESPAÑA No. 63 pp. 39-64. (http://redalyc.uaemex.mx/pdf/174/17412307002.pdf) 04-17-2012

Fillis I. (2001) "Small firms internationatilisation: an investigative survey and future research directions" Management Decision Vol. 39, No. 9 pp. 767-783 
Flor Peris, M. L.Oltra Mestre, M.J. (2009) "La estrategia exportadora de la empresa y su relación con el resultado internacional” Investigaciones Europeas de Dirección y Economía de la Empresa. Vol. 16, No. 1, 2010, pp. 15-29

Galván Sánchez I (2003) La formación de la estrategia de selección de mercados exteriores en el proceso de internacionalización de las empresas. Tesis Doctoral Universidad de Las Palmas de Gran Canaria, España (http://www.eumed.net/ tesis/igs/index.htm )

Gómez, M. y Valenzuela, A. (1999). Las capacidades organizativas y los mercados internacionales: Una visión interdisciplinar desde las teorías de la organización y del marketing. Revista Encuentros Multidisciplinares, Madrid. Vol. 2, No. 6. Pp. 1-11

Gómez Édgar y González Gustavo (2011) "Proceso de internacionalización de empresas del Área Metropolitana de Bucaramanga" Revista Lebret No. 3, Universidad Santo Tomás, Bucaramanga.

Gheorghiu M. C. (2007) Factores de marketing que determinan el rendimiento internacional de las empresas exportadoras españolas: enfoque sobre las empresas born-global Tesis Universidad Autónoma de Barcelona.( http://idem. uab. es/treballs\%20recerca/Maria\%20Gheorghiu.pdf )

Hodge, B. J.; Anthony W. P. y Gales, L. M. (1998) Teoría de la organización: un enfoque estratégico. $5^{\text {a }}$. Ed. España: Editorial Prentice Hall

Hymer, S. (1960), The International operations of national firms: a study of foreign direct investment, Ph. Dissertation, MIT Press, Cambridge. (http://teaching.ust. hk/ mgto650p/meyer/readings/1/01_Hymer.pdf )

Iborra, M., Menguzzato, M. y Ripollés, M. (1998). Creación de empresas internacionales: Redes informales y obtención de recursos. Revista Europea de Dirección y Economía de la Empresa. Vol. 7, No. 3, pp. 147-160

Jacobsohn G. (2004) La creación de nuevas firmas: Aportes de la dirección estratégica Pensamiento \& Gestión, 16. Universidad del Norte, 135-157

Jiménez Palmero A. (2008) “La Internacionalización de la Empresa a través del Enfoque de Recursos y Capacidades" Gestión Joven Revista de la Agrupación Joven Iberoamericana de Contabilidad y Administración de Empresas No. 2 Octubre 2008

Johanson, J., y Vahlne, J. -E. (1977), “The internationalization of the firm-A Model of knowledge development and increasing foreign market commitmnents", Journalof International Business Studies, Vol. 8 (1), pp. 23-32 
Jovell L. (1990) Estrategia empresarial e innovación como concluyentes de la intensidad exportadora: "Un análisis empírico" Tesis Doctoral Universidad Ramón Llull, Barcelona, España.

(http://www.tesisenred.net/bitstream/handle/10803/9312/JovellTurro. pdf?sequence $=1$ )

Katsikeas, Constantine S., Leonidas C. Leonidou, y Neil A. Morgan (2000) Firmlevel export performance assessment: Review, evaluation, and development. Journal of the Academy of Marketing Science 28 (4):493-511

Leonidou, L., Katsikeas, C., y Samiee, S. (2002). Marketing strategy determinants of export performance: a meta-analysis. Journal of Business Research, 55(1), 51-67

Londoño-Ávila A (2012) Internacionalización de la empresa colombiana Cementos Argos: una mirada desde diferentes teorías Revista de Negocios Internacionales. Vol. 5. No. 1 - Enero - Junio de 2012 - Pp. 29 - 43

Losada Pérez et al. (2006) "La performance de exportación: revisión teórica y propuesta de una marco integrador" Investigaciones Europeas de Dirección y Economía de la Empresa, Vol. 12 No. 1 pp. 85-105 (http://www.aedem-virtual. com/articulos/iedee/v12/121085.pdf ) 04-09-2012

Madsen, T. K. (1987) "Empirical export performance studies: A review of conceptualizations and findings" Advances in International Marketing, Vol. 2, Ed. S. T. Cavusgil and C. Axinn, 177-198.

Martínez Carazo, P C (2009) Proceso del desarrollo exportador de la PYME colombiana Cuadernos de Gestión, Vol. 9, No. 1, 2009, pp. 47-65 Universidad del País Vasco, España

Medina Muñoz D. R (1998) Una visión integral de la empresa basada en los recursos, el conocimiento y el aprendizaje. Investigaciones Europeas de Dirección y Economía de la Empresa. Vol. 4, W2, pp. 77-90 (http://www.aedem-virtual.com/ articulos/iedee/v04/042077.pdf ) 02-03-2012

Mesa Callejas R. J. y Pérez Robles S. P. (2011) Desempeño exportador de Antioquia y marco institucional para su desarrollo: el papel de la Mipyme* Perfil de Coyuntura Económica No. 17, agosto 2011, pp. 123-139. Universidad de Antioquia

Milesi D. et al. (2007) Desarrollo de ventajas competitivas: pymes exportadoras exitosas en Argentina, Chile y Colombia. Revista de la CEPAL 92. Santiago

Moen, O. y Servais M. (2002). Born Global or Gradual Global? Examining the Export Behavior of small and Medium-Sized Enterprises. Journal of International Marketing, Vol. 10, No. 3, pp. 49-72

Moghaddam, F. M. y otros. (2011) “The Influence of Export Marketing Strategy Determinants on Firm Export Performance: A Review of Empirical literatures 
Between 1993-2010" International Journal of Fundamental Psychology \& Social Sciences, Vol. 1, No. 2, pp. 26-34, Dec. (http:/fundamentaljournals.org/ijfpss/ downloads/7-IJFPSS\%20\%28Moghadam\% 20061211\%29\%2826-34\%29.pdf )

Monreal J. (2009) Análisis del comportamiento exportador de la empresa española desde el enfoque de los recursos y capacidades. Tesis Doctoral Facultad de Economía y Empresa Universidad de Murcia (http://www.tesisenred.net/handle/ 10803/11003)

Moori Koering V. y otros (2006) Perfil de la Pyme exportadora exitosa el caso colombiano FUNDES, Bogotá

Moreno O. (2007) El marketing internacional como herramienta indispensable para una exportación efectiva. Ciencia en su PC, No. 2, 2007, pp. 1-10. Instituto de Información Científica y Tecnológica Santiago de Cuba, http://redalyc. uaemex. $\mathrm{mx} / \mathrm{redalyc} / \mathrm{pdf} / 1813 / 181320217005 . \mathrm{pdf}$

Niron H. y Tamar A. (2002) Small and Medium Sized Multinationals: The Internationalization Process of Born Global Companies Working Paper No. 02/22Bradford University School of Management. (http://www.brad.ac.uk/acad/ management/external/pdf/workingpapers/Booklet_02-22.pdf)

Ohmae K (1990) El poder de la Triada Panorama de la competencia mundial de la próxima década, McGraw Hill, México

Ohmae K. (2005) El próximo escenario global Desafíos y oportunidades en un mundo sin fronteras, Bogotá, Norma.

Porter M (1990) La ventaja competitiva de las naciones, Editorial Vergara, Buenos Aires.

Rialp, A. (1997), "Las fases iniciales del proceso de internacionalización de las empresas industriales catalanas: una aproximación empírica”, Tesis Doctoral, Universidad Autónoma de Barcelona (http://www.tdx.cat/TDX-0331108-122058/)

Rialp, A. (1999), "Los Enfoques Micro-organizativos de la Internacionalización de la Empresa: Una Revisión y Síntesis de la Literatura”, Información Comercial Española(ICE), Octubre 1999, No. 781, pp. 117-128

Sánchez-Meca, J. (1999) “Metaanálisis para la Investigación Científica”, en Sarabia Sánchez, F.J. (1999) “Metodología para la Investigación en Marketing y Dirección de Empresas”, Ediciones Pirámide, Madrid

Sinisterra M.M. (2009) Determinantes de la multi-localización de empresas exportadoras de manufacturas en Colombia. Lecturas de Economía, 71 (juliodiciembre), pp. 77-106. Universidad de Antioquia 
Sousa, Carlos M. P. (2004) Export performance measurement: An evaluation of theempirical research in the literature. Academy of Marketing Science Review (http://amsreview.org/articles/sousa09-2004.pdf)

Sousa, C.M.P., Martínez-López, F.J., and Coelho, F. (2008) "The determinants of export performance: a review of the research in the literature between 1998 and 2005" International Journal of Management Review 10 (4), 343-374.

Valencia Rodríguez (2009). La competitividad, un estudio en las Pymes desde la gestión del conocimiento Libre Empresa, 11 9-21

Vázquez N. y Vázquez L. (2007) 'Principales enfoques teóricos e investigaciones empíricas generales sobre la internacionalización de PYMES: un estudio exploratorio de investigaciones entre 1999 y 2004" E-journal UNAM No. 222 mayo-agosto 2007: 41-57 (http://www.ejournal.unam.mx/rca/222/ RCA000022204. pdf consulta 11-11-2011)

Vernon, R. (1966), "International investment and international trade in the product cycle”, Quarterly Journal of Economics, May, pp. 190-207

Villareal O. (2005) "La internacionalización de la empresa y la empresa multinacional: una revisión conceptual contemporánea" Cuadernos de Gestión. Vol. 5. No. 2 (Año 2005), pp. 55-73

Villarreal O (2006) La estrategia de internacionalización de la empresa: un estudio de casos de multinacionales vascas. Tesis Doctoral Universidad del País Vasco. Dos tomos. http://www.aleive.net/2012/02/la-estrategia-de-internacionalizacion. html

Villarreal, O y Landeta J. (2010) "El estudio de casos como metodología de investigación científica en dirección y economía de la empresa: una aplicación a la internacionalización" Investigaciones Europeas de Dirección y Economía de la Empresa, Vol. 16 No. 3, 2010, pp. 31-52, (http://www.aedem-virtual.com/ articulos/ iedee/v16/163031.pdf) 04-05-12

Welch, L.S., y Luostarinen, R. (1988), “Internationalisation: Evolution of a Concept", Journal of General Management, Vol. 14 (2), pp. 34-55

Wernerfelt, B. (1984): “A Resource-Based View of the firm”, Strategic Management Journal, 5(2): 171-180

Zou, S y S Stand (1998). The determinants of export performance: A review of the empirical literature between 1987 and 1997. International Marketing Review 15 (5):333-356. 